\title{
Sparse grid high-order ADI scheme for option pricing in stochastic volatility models
}

\author{
Bertram Düring* Christian Hendricks ${ }^{\dagger} \quad$ James Miles $^{\ddagger}$
}

March 27, 2018

\begin{abstract}
We present a sparse grid high-order alternating direction implicit (ADI) scheme for option pricing in stochastic volatility models. The scheme is second-order in time and fourth-order in space. Numerical experiments confirm the computational efficiency gains achieved by the sparse grid combination technique.
\end{abstract}

\section{Introduction}

Stochastic volatility models such as the Heston model 22] have become one of the standard approaches in financial option pricing. For some stochastic volatility models and under additional restrictions, closed-form solutions can be obtained by Fourier methods (e.g. 22, [15]). Another approach is to derive approximate analytic expressions, see e.g. 22 and the literature cited therein. In general, however, - even in the Heston model 22 when the parameters in it are non constant - the partial differential equations arising from stochastic volatility models have to be solved numerically.

In the mathematical literature, there are many papers on numerical methods for option pricing, mostly addressing the one-dimensional case of a single risk factor and using standard, second order finite difference methods (see, e.g., 41 and the references therein). More recently, high-order finite difference schemes (fourth order in space) were proposed [19, 37, 40, that use a compact stencil (three points in space). In the option pricing context, see e.g. [11, 12, 30].

There are less works considering numerical methods for option pricing in stochastic volatility models, i.e., for two spatial dimensions. Finite difference approaches that are used are often standard, second-order methods, e.g. in [28] where different efficient methods for solving the American option pricing problem for the Heston model are proposed. In [9] a high-order compact finite difference scheme for option pricing in the Heston model is derived and this approach is extended to non-uniform grids in [10. Other approaches include

\footnotetext{
*Department of Mathematics, University of Sussex, Pevensey II, Brighton, BN1 9QH, United Kingdom, bd80@sussex.ac.uk

${ }^{\dagger}$ Chair of Applied Mathematics / Numerical Analysis, Bergische Universität Wuppertal, Gaußstraße 20, 42097 Wuppertal, Germany, hendricks@uni-wuppertal.de

${ }^{\ddagger}$ Department of Mathematics, University of Sussex, Pevensey II, Brighton, BN1 9QH, United Kingdom, james.miles@sussex.ac.uk
} 
finite element-finite volume [4], multigrid [5], sparse wavelet [27, FFT-based [36] or spectral methods [33.

The classical alternating direction implicit (ADI) method, introduced by Peaceman and Rachford [34, Douglas [6, 7], Fairweather and Mitchell [33, is a very powerful method that is especially useful for solving parabolic equations (without mixed derivative terms) on rectangular domains. Beam and Warming [1, however, have shown that no simple ADI scheme involving only discrete solutions at time levels $n$ and $n+1$ can be second-order accurate in time in the presence of mixed derivatives. To overcome this limitation, unconditionally stable ADI schemes which are second order in time have been proposed by Hundsdorfer and Verwer 26, 25, and more recently by in't Hout and Welfert 24. These schemes are second-order accurate in time and space. In 23 different second-order ADI schemes of this type are applied to the Heston model. In 13 this approach is combined with different high-order discretisations in space, using high-order compact schemes for two-dimensional convection-diffusion problems with mixed derivatives and constant coefficients. In 21] this approach is combined with sparse grids and applied to multi-dimensional diffusion equations, again with constant coefficients. Building on the ideas in [26, 25, 13], a high-order (second-order accurate in time and fourth-order accurate in space) ADI method for option pricing in stochastic volatility models which involve the solution of two-dimensional convection-diffusion equations with mixed derivative terms and space-dependent coefficients is derived in 16.

In this chapter we combine the approaches from [21] and [16, to obtain a sparse grid high-order ADI scheme for option pricing in stochastic volatility models. In the next section we recall stochastic volatility models for option pricing and the related convection-diffusion partial differential equations. Section 3 is devoted to the Hundsdorfer-Verwer ADI splitting in time. The spatial discretisation is introduced in Section 4 for the implicit steps, and in Section 5 for the explicit steps. The solution of the resulting scheme and the discretisation of boundary conditions are discussed in Sections 6 and 7 . The sparse grid combination technique is explained in Section 8. We present numerical convergence results in Section 9 ,

\section{Stochastic volatility models}

We consider the following class of stochastic volatility models: assume that asset spot price $0 \leq S(t)<\infty$ and variance $0 \leq \sigma(t)<\infty$ follow two stochastic diffusive processes for $t \in[0, T]$,

$$
\begin{aligned}
& d S(t)=\mu S(t) d t+\sqrt{\sigma(t)} S(t) d W^{(1)}(t), \\
& d \sigma(t)=\kappa(\sigma(t))^{\alpha}(\theta-\sigma(t)) d t+v(\sigma(t))^{\beta} d W^{(2)}(t),
\end{aligned}
$$

which are characterised by two Brownian motions, $d W^{(1)}(t)$ and $d W^{(2)}(t)$, with constant correlation parameter $d W^{(1)}(t) d W^{(2)}(t)=\rho d t$. The drift coefficient for stochastic asset returns is given by the mean return of the asset where $\mu \in \mathbb{R}$ and the diffusion coefficient is given by $\sqrt{\sigma(t)} S(t)$.

The drift coefficient of the asset variance is given by $\kappa(\sigma(t))^{\alpha}(\widetilde{\theta}-\sigma(t))$, where constants $\kappa \geq 0$ and $\theta \geq 0$ are the mean reversion speed of $\sigma(t)$ and the long run mean of $\sigma(t)$, respectively. The diffusion coefficient is given by $v(\sigma(t))^{\beta}$ where 
constant $v \geq 0$ is the volatility of volatility. The constant riskless interest rate is denoted by $r \geq 0$. The constants $\alpha, \beta$ determine the stochastic volatility model used.

The class of stochastic volatility models (1) includes a number of known stochastic volatility models: The most prominent stochastic volatility model, the Heston model 22] (also called square root (SQR) model) specifies the variance by

$$
d \sigma(t)=\kappa(\theta-\sigma(t)) \mathrm{d} t+v \sqrt{\sigma(t)} \mathrm{d} W^{(2)}(t) .
$$

Other known stochastic volatility models include the GARCH (or VAR model) model, see [8, where the stochastic variance is modelled by

$$
d \sigma(t)=\kappa(\theta-\sigma(t)) \mathrm{d} t+v \sigma(t) \mathrm{d} W^{(2)}(t),
$$

and the $3 / 2$ model 32 in which the variance follows the process

$$
d \sigma(t)=\kappa(\theta-\sigma(t)) \mathrm{d} t+v \sigma^{\frac{3}{2}}(t) \mathrm{d} W^{(2)}(t) .
$$

All of the three stochastic volatility models mentioned above use a linear meanreverting drift for the stochastic process of the variance $v(t)$, but there are also models, in which the drift is mean reverting in a non-linear fashion. Following [4, we denote these models with an additional "N": in the $S Q R N$ model the stochastic variance follows

$$
d \sigma(t)=\kappa \sigma(t)(\theta-\sigma(t)) \mathrm{d} t+v \sqrt{\sigma(t)} \mathrm{d} W^{(2)}(t),
$$

in the VARN model

$$
d \sigma(t)=\kappa \sigma(t)(\theta-\sigma(t)) \mathrm{d} t+v \sigma(t) \mathrm{d} W^{(2)}(t)
$$

and in the $3 / 2-N$ model

$$
d \sigma(t)=\kappa \sigma(t)(\theta-\sigma(t)) \mathrm{d} t+v \sigma^{\frac{3}{2}}(t) \mathrm{d} W^{(2)}(t),
$$

see 4 .

Applying standards arbitrage arguments and Itos lemma to the class of stochastic volatility models (1), we can derive the following second order partial differential equation for any financial derivative $V(S, \sigma, t)$, to be solved backwards in time with $0<S<\infty, 0<\sigma<\infty, t \in[0, T)$ :

$V_{t}+\frac{S^{2} \sigma}{2} V_{S S}+\rho v \sigma^{\beta+\frac{1}{2}} S V_{S \sigma}+\frac{v^{2} \sigma^{2 \beta}}{2} V_{\sigma \sigma}+r S V_{s}+\left[\kappa \sigma^{\alpha}(\theta-\sigma)-\lambda_{0} \sigma\right] V_{\sigma}-r V=0$.

Here, $\lambda_{0} \sigma(t)$ is the market price of volatility risk, where $\lambda_{0} \in \mathbb{R}$, which is usually assumed to be proportional to the variance. In the following we assume $\lambda_{0}=0$ for streamlining the presentation. The generalisation to the case $\lambda_{0} \neq 0$ is straightforward by consistently adding in the additional term in the coefficient of $V_{\sigma}$. The boundary conditions and final condition are determined by the type of financial derivative $V(S, \sigma, t)$ we are solving for. The boundary conditions of any European option will depend on a prescribed exercise price, denoted here 
by $E>0$. For example, in the case of the European Put Option:

$$
\begin{aligned}
V(S, \sigma, T) & =\max (E-S, 0), & & 0<S<\infty, 0<\sigma<\infty, \\
\lim _{S \rightarrow \infty} V(S, \sigma, t) & =0, & & 0<\sigma<\infty, 0<t<T, \\
V(0, \sigma, t) & =E \exp (-r(T-t)), & & 0<\sigma<\infty, 0<t<T, \\
\lim _{\sigma \rightarrow \infty} V_{\sigma}(S, \sigma, t) & =0, & & 0<S<\infty, 0<t<T,
\end{aligned}
$$

The remaining boundary condition at $\sigma=0$ can be obtained by looking at the formal limit $\sigma \rightarrow 0$ in 2 , i.e.,

$$
V_{t}+r S V_{S}+\kappa \theta V_{\sigma}-r V=0, \quad T>t \geq 0, S>0, \text { as } \sigma \rightarrow 0 .
$$

This boundary condition is used frequently, e.g. in [28, 44. Alternatively, one can use a homogeneous Neumann condition [5], i.e.,

$$
V_{\sigma}(S, 0, t)=0, \quad 0<S<\infty, 0<t<T .
$$

By using a change of variables:

$$
x=\ln \frac{S}{E}, \quad y=\frac{\sigma}{v}, \quad \tau=T-t, \quad u=\exp (r \tau) \frac{V}{E}
$$

we transform the partial differential equation to an convection-diffusion equation in two spatial dimensions with a mixed derivative term. The transformed partial differential equation and boundary/initial conditions are now satisfied by $u(x, y, \tau)$, where $x \in \mathbb{R}, y>0, \tau \in(0, T]$ :

$u_{\tau}=\frac{v y}{2} u_{x x}+\frac{(v y)^{2 \beta}}{2} u_{y y}+\rho(v y)^{\beta+\frac{1}{2}} u_{x y}+\left(r-\frac{v y}{2}\right) u_{x}+\left(\kappa(v y)^{\alpha} \frac{\theta-v y}{v}\right) u_{y}$,

$$
\begin{aligned}
u(x, y, 0) & =\max (1-\exp (x), 0), & -\infty<x<\infty, 0<y<\infty, \\
\lim _{x \rightarrow \infty} u(x, y, \tau) & =0, & 0<y<\infty, 0 \leq \tau<T, \\
\lim _{x \rightarrow-\infty} u(x, y, \tau) & =1, & 0<y<\infty, 0 \leq \tau<T, \\
\lim _{y \rightarrow \infty} u_{y}(x, y, \tau) & =0, & -\infty<x<\infty, 0<\tau \leq T, \\
\lim _{y \rightarrow 0} u_{y}(x, y, \tau) & =0, & -\infty<x<\infty, 0<\tau \leq T .
\end{aligned}
$$

In order to discretise the problem and solve numerically, we truncate our spatial boundaries to finite values. Take $L_{1} \leq x \leq K_{1}$, where $L_{1}<K_{1}$, and $L_{2} \leq y \leq$ $K_{2}$, where $0<L_{2}<K_{2}$, so that the spatial domain forms a closed rectangle in $\mathbb{R}^{2}$ of $M \times N$ points with uniform spacing of $\Delta_{x}$ in the $x$-direction and $\Delta_{y}$ in the $y$-direction:

$x_{i}=L_{1}+(i-1) \Delta_{x}, i=1,2, \ldots, M, \quad y_{j}=L_{2}+(j-1) \Delta_{y}, j=1,2, \ldots, N$.

The lower $y$-boundary is truncated to $L_{2}>0$ to ensure non-degeneracy of the partial differential equation for all values of $y$. We assume cell aspect ratios to be moderate. We also take a uniform partition of $\tau \in[0, T]$ into $P$ points such that $\tau_{k}=(k-1) \Delta_{\tau}$, where $k=1,2, \ldots, P$. We denote the discrete approximation of $u\left((i-1) \Delta_{x},(j-1) \Delta_{y},(k-1) \Delta_{\tau}\right)$ by $u_{i, j}^{k}$ and $U^{n}=\left(u_{i, j}^{n}\right)_{i, j}$. 


\section{Hundsdorfer-Verwer ADI splitting scheme}

We consider the Alternating Direction Implicit (ADI) time-stepping numerical method proposed by Hundsdorfer and Verwer [26, 25]. Our partial differential equation (5) takes the form $u_{\tau}=F(u)$. We employ the splitting $F(u)=F_{0}(u)+$ $F_{1}(u)+F_{2}(u)$ where unidirectional and mixed derivative differential operators are given by:

$$
\begin{aligned}
F_{0}(u)=\rho(v y)^{\beta+\frac{1}{2}} u_{x y}, F_{1}(u) & =\frac{v y}{2} u_{x x}+\left(r-\frac{v y}{2}\right) u_{x}, \\
& F_{2}(u)=\frac{(v y)^{2 \beta}}{2} u_{y y}+\left(\kappa(v y)^{\alpha} \frac{\theta-v y}{v}\right) u_{y} .
\end{aligned}
$$

We consider (5) with the splitting (7) and look for a semi-discrete approximation $U^{n} \approx u\left(\tau_{n}\right)$ at time $n \Delta_{\tau}$. Given an approximation $U^{n-1}$ we can calculate an approximation for $U^{n}$ at time $n \Delta_{\tau}$ using the differential operators from (7):

$$
\begin{aligned}
Y_{0} & =U^{n-1}+\Delta_{t} F\left(U^{n-1}\right), \\
Y_{1} & =Y_{0}+\phi \Delta_{t}\left(F_{1}\left(Y_{1}\right)-F_{1}\left(U^{n-1}\right)\right), \\
Y_{2} & =Y_{1}+\phi \Delta_{t}\left(F_{2}\left(Y_{2}\right)-F_{2}\left(U^{n-1}\right)\right), \\
\widetilde{Y}_{0} & =Y_{0}+\psi \Delta_{t}\left(F\left(Y_{2}\right)-F\left(U^{n-1}\right)\right), \\
\widetilde{Y}_{1} & =\widetilde{Y}_{0}+\phi \Delta_{t}\left(F_{1}\left(\widetilde{Y}_{1}\right)-F_{1}\left(Y_{2}\right)\right), \\
\tilde{Y}_{2} & =\widetilde{Y}_{1}+\phi \Delta_{t}\left(F_{2}\left(\widetilde{Y}_{2}\right)-F_{2}\left(Y_{2}\right)\right), \\
U^{n} & =\widetilde{Y}_{2} .
\end{aligned}
$$

The parameter $\psi$ is taken to be $\psi=1 / 2$ to ensure second-order accuracy in time. The parameter $\phi$ is typically fixed to $\phi=1 / 2$. Larger values give stronger damping of the implicit terms while lower values return better accuracy. The role of $\phi$ is discussed in [26]. Its influence in the connection with high-order spatial approximations is investigated numerically in [16.

The first and fourth step in (8) can be solved explicitly, while the remaining steps are solved implicitly. Our aim is to derive high-order spatial discretisations of the differential operators. Following [13] we combine high-order compact finite difference methods for the implicit steps with a (classical, non-compact) high-order stencil for the explicit steps.

\section{High-order compact scheme for implicit steps}

For $F_{1}(u)$, consider the one-dimensional convection-diffusion equation

$$
u_{x x}+c_{1} u_{x}=c_{2} g
$$

with constants $c_{1}=2 r /(v y)-1$ and $c_{2}=2 /(v y)$. To discretise the partial derivatives in $(9)$, we employ standard, centered second-order finite difference operators, denoted by $\delta_{x 0}$ and $\delta_{x}^{2}$. The second-order terms in the truncation error involve higher-order partial derivatives, $u_{x x x}$ and $u_{x x x x}$. Hence, if we can find second-order accurate expressions for $u_{x x x}$ and $u_{x x x x}$, using only information on the compact stencil, then it will be possible to approximate $u_{x}$ and $u_{x x}$ 
with fourth order accuracy on the compact stencil. By differentiating 90 once and twice with respect to $x$, respectively, it is possible to express $u_{x x x}$ and $u_{x x x x}$ in terms of first- and second-order derivatives of $u$ and $g$ with respect to $x$. We obtain the following relations, concisely written in matrix form,

$$
\left[\begin{array}{cccc}
1 & 0 & \frac{1}{6} & 0 \\
0 & 1 & 0 & \frac{1}{12} \\
0 & c_{1} \Delta_{x}^{2} & 1 & 0 \\
0 & 0 & c_{1} & 1
\end{array}\right]\left[\begin{array}{c}
u_{x} \\
u_{x x} \\
\Delta_{x}^{2} u_{x x x} \\
\Delta_{x}^{2} u_{x x x x}
\end{array}\right]=\left[\begin{array}{c}
\delta_{x 0} u_{i, j} \\
\delta_{x}^{2} u_{i, j} \\
c_{2} \Delta_{x}^{2} g_{x} \\
c_{2} \Delta_{x}^{2} g_{x x}
\end{array}\right]+\left[\begin{array}{c}
\mathcal{O}\left(\Delta_{x}^{4}\right) \\
\mathcal{O}\left(\Delta_{x}^{4}\right) \\
0 \\
0
\end{array}\right]=\left[\begin{array}{c}
\delta_{x 0} u_{i, j} \\
\delta_{x}^{2} u_{i, j} \\
c_{2} \Delta_{x}^{2} \delta_{x 0} g_{i, j} \\
c_{2} \Delta_{x}^{2} \delta_{x}^{2} g_{i, j}
\end{array}\right]+\mathcal{O}\left(\Delta_{x}^{4}\right) .
$$

This shows that only second-order approximations for $u_{x}, u_{x x}, g_{x}$ and $g_{x x}$ are needed. Using these relations to discretise (9) and to replace the partial derivatives $u_{x x x}$ and $u_{x x x x}$ in the truncation error, yields a fourth-order compact approximation for $(9)$ at all points of the spatial grid except those that lie on the $x$ - and $y$-boundaries. We refer to 16 for more details of the derivation of the compact high-order spatial discretisation.

To approximate $F_{1}(u)$ at points along the $x$ boundaries of the inner grid of the spatial domain, we will require a contribution from the Dirichlet values at the $x$-boundaries of the spatial domain. We collect these separately in a vector $d$. Details on the boundary conditions are given in Section 7. The resulting linear system to be solved can be written in matrix form:

$$
A_{x} u=B_{x} g+d,
$$

where $u=\left(u_{2,2}, u_{2,3}, \ldots, u_{M-1, N-1}\right), g=\left(g_{2,2}, g_{2,3}, \ldots, g_{M-1, N-1}\right)$. The coefficient matrices $A_{x}$ and $B_{x}$ are block diagonal matrices, with the following structure:

$A_{x}=\left[\begin{array}{cccc}A_{x}^{1,1} & 0 & 0 & 0 \\ 0 & A_{x}^{2,2} & 0 & 0 \\ 0 & 0 & \ddots & 0 \\ 0 & 0 & 0 & A_{x}^{N-2, N-2}\end{array}\right], \quad B_{x}=\left[\begin{array}{cccc}B_{x}^{1,1} & 0 & 0 & 0 \\ 0 & B_{x}^{2,2} & 0 & 0 \\ 0 & 0 & \ddots & 0 \\ 0 & 0 & 0 & B_{x}^{N-2, N-2}\end{array}\right]$,

where each $A_{x}^{j, j}=\operatorname{diag}\left[a_{-1}^{j, j}, a_{0}^{j, j}, a_{1}^{j, j}\right]$ and $B_{x}^{j, j}=\operatorname{diag}\left[b_{-1}^{j, j}, b_{0}^{j, j}, b_{1}^{j, j}\right]$ are tridiagonal matrices. Explicit expression for all coefficients are given in [16].

For $F_{2}(u)$ the derivation can be presented in a concise form, similar as for $F_{1}(u)$, again we refer to 16 for additional details. Consider the one-dimensional convection-diffusion equation

$$
u_{y y}+c_{1} u_{y}=c_{2} g
$$

with $c_{1}(y)=2 \kappa(v y)^{\alpha-2 \beta}(\theta-v y) / v$ and $c_{2}(y)=2 /(v y)^{2 \beta}$, the necessary relations can be concisely written in matrix form,

$$
\begin{gathered}
{\left[\begin{array}{cccc}
1 & 0 & \frac{1}{6} & 0 \\
0 & 1 & 0 & \frac{1}{12} \\
c_{1}^{\prime} \Delta_{y}^{2} & c_{1} \Delta_{y}^{2} & 1 & 0 \\
c_{1}^{\prime \prime} \Delta_{y}^{2} & 2 c_{1}^{\prime} \Delta_{y}^{2} & c_{1} & 1
\end{array}\right]\left[\begin{array}{c}
u_{y} \\
u_{y y} \\
\Delta_{y}^{2} u_{y y y} \\
\Delta_{y}^{2} u_{y y y}
\end{array}\right]} \\
=\left[\begin{array}{c}
\delta_{y 0} u_{i, j} \\
\delta_{y}^{2} u_{i, j} \\
\Delta_{y}^{2}\left(\delta_{y}^{2} c_{2, j} g_{i, j}+2 \delta_{y 0} c_{2, j} \delta_{y 0} g_{i, j}+c_{2, j} \delta_{y}^{2} g_{i, j}\right)
\end{array}\right]+\mathcal{O}\left(\Delta_{y}^{4}\right),
\end{gathered}
$$


where the first two lines of the system correspond to standard, central secondorder difference approximations, while the third and fourth are obtained from the repeated differentiation of $(10)$. Using these relations to discretise $(10)$ and to replace the partial derivatives $u_{y y y}$ and $u_{y y y}$ in the truncation error, yields a fourth-order compact approximation for 10 .

We obtain a linear system which can be represented in matrix form:

$$
A_{y} u=B_{y} g
$$

where $u=\left(u_{2,2}, u_{2,3}, \ldots, u_{M-1, N-1}\right), g=\left(g_{2,2}, g_{2,3}, \ldots, g_{M-1, N-1}\right)$. We do not impose any boundary conditions in $y$-direction, but discretise the boundary grid points with the same scheme, and handle resulting ghost points via extrapolation; details on the boundary conditions are given in Section 7. The coefficient matrices $A_{y}$ and $B_{y}$ are block tri-diagonal matrices with the following structures:

$$
\begin{aligned}
\mathbf{A}_{\mathbf{y}}= & {\left[\begin{array}{ccccc}
A_{y}^{1,1} & A_{y}^{1,2} & 0 & 0 & 0 \\
A_{y}^{2,1} & A_{y}^{2,2} & A_{y}^{2,3} & 0 & 0 \\
0 & \ddots & \ddots & \ddots & 0 \\
0 & 0 & A_{y}^{N-3, N-4} & A_{y}^{N-3, N-3} & A_{y}^{N-3, N-2} \\
0 & 0 & 0 & A_{y}^{N-2, N-3} & A_{y}^{N-2, N-2}
\end{array}\right], } \\
\mathbf{B}_{\mathbf{y}}= & {\left[\begin{array}{ccccc}
B_{y}^{1,1} & B_{y}^{1,2} & 0 & 0 & 0 \\
B_{y}^{2,1} & B_{y}^{2,2} & B_{y}^{2,3} & 0 & 0 \\
0 & \ddots & \ddots & \ddots & 0 \\
0 & 0 & B_{y}^{N-3, N-4} & B_{y}^{N-3, N-3} & B_{y}^{N-3, N-2} \\
0 & 0 & 0 & B_{y}^{N-2, N-3} & B_{y}^{N-2, N-2}
\end{array}\right], }
\end{aligned}
$$

where each $A_{y}^{j, j}=\operatorname{diag}\left[a^{i, j}\right]$ and $B_{y}^{j, j}=\operatorname{diag}\left[b^{i, j}\right]$ are diagonal matrices. Explicit expression for all coefficients are given in [16.

\section{High-order scheme for explicit steps}

The first and fourth steps of the ADI scheme (8) operate only on previous approximations to explicitly calculate an updated approximation. The differential operator in these steps takes the form of the right hand side of (5). For the mixed derivative term it seems not to be possible to exploit the structure of the differential operator to obtain a fourth-order approximation on a compact computational stencil. Hence, in order to maintain fourth-order accuracy of the scheme in the explicit steps of (8), the derivatives in each differential operator $F_{0}, F_{1}$ and $F_{2}$ are approximated using classical, fourth-order central difference operators which operate on a larger $5 \times 5$-stencil in the spatial domain. Here we use the shift operator defined by:

$$
s_{x}=e^{\Delta_{x} \delta_{x}} \text { where }\left(s_{x} u\right)_{i, j}=u_{i+1, j}, \quad s_{y}=e^{\Delta_{y} \delta_{y}} \text { where }\left(s_{y} u\right)_{i, j}=u_{i, j+1} .
$$


For $F_{1}(u)=\frac{v y}{2} u_{x x}-\left(\frac{v y}{2}-r\right) u_{x}$, we have the following scheme:

$$
\begin{aligned}
& {\left[\frac{v y}{2} u_{x x}+\left(r-\frac{v y}{2}\right) u_{x}\right]_{i, j} }=\frac{v y_{j}}{2}\left(\frac{-s_{x}^{-2}+16 s_{x}^{-1}-30+16 s_{x}-s_{x}^{2}}{12 \Delta_{x}^{2}}\right) u_{i, j} \\
&+\left(r-\frac{v y_{j}}{2}\right)\left(\frac{s_{x}^{-2}-8 s_{x}^{-1}+8 s_{x}-s_{x}^{2}}{12 \Delta_{x}}\right) u_{i, j}+\mathcal{O}\left(\Delta_{x}^{4}\right)
\end{aligned}
$$

For $F_{2}(u)=\frac{(v y)^{2 \beta}}{2} u_{y y}+\frac{\kappa(v y)^{\alpha}(\theta-v y)}{v} u_{y}$, we have:

$$
\begin{aligned}
{\left[\frac{(v y)^{2 \beta}}{2} u_{y y}+\right.} & \left.\frac{\kappa(v y)^{\alpha}(\theta-v y)}{v} u_{y}\right]_{i, j} \\
= & \frac{\left(v y_{j}\right)^{2 \beta}}{2}\left(\frac{-s_{y}^{-2}+16 s_{y}^{-1}-30+16 s_{y}-s_{y}^{2}}{12 \Delta_{y}^{2}}\right) u_{i, j} \\
& +\frac{\kappa\left(v y_{j}\right)^{\alpha}\left(\theta-v y_{j}\right)}{v}\left(\frac{s_{y}^{-2}-8 s_{y}^{-1}+8 s_{y}-s_{y}^{2}}{12 \Delta_{y}}\right) u_{i, j}+\mathcal{O}\left(\Delta_{y}^{4}\right)
\end{aligned}
$$

Finally, for the mixed derivative term $F_{0}=\rho(v y)^{\beta+\frac{1}{2}} u_{x y}$, the following computational stencil is used:

$$
\begin{aligned}
& {\left[\rho(v y)^{\beta+\frac{1}{2}} u_{x y}\right]_{i, j}} \\
& =\rho\left(v y_{j}\right)^{\beta+\frac{1}{2}}\left(\frac{s_{x}^{-2}-8 s_{x}^{-1}+8 s_{x}-s_{x}^{2}}{12 \Delta_{x}}\right)\left(\frac{s_{y}^{-2}-8 s_{y}^{-1}+8 s_{y}-s_{y}^{2}}{12 \Delta_{y}}\right) u_{i, j} \\
& +\mathcal{O}\left(\Delta_{x}^{4} \Delta_{y}^{4}\right)+\mathcal{O}\left(\Delta_{x}^{4}\right)+\mathcal{O}\left(\Delta_{y}^{4}\right) .
\end{aligned}
$$

Using these fourth-order approximations, the first and fourth step in 8 can be computed directly. The values at the spatial boundaries for each solution of the ADI scheme are determined by the boundary conditions, the computational stencil is required for all remaining points in the spatial domain. For the explicit steps, the $5 \times 5$-point computational stencil exceeds the spatial boundary when we wish to approximate differential operator $F(u)$ at any point along the boundary of the spatial domain's inner grid. For example if we wish to evaluate $F\left(u_{2,2}\right)$, we will require contributions from ghost points which fall outside the spatial domain, as marked by bullet points in Figure 1. We extrapolate information from grid points $u\left(x_{i}, y_{j}\right)$, where $i=1, \ldots, M-1, j=1, \ldots, N-1$ to establish values at these ghost points for the purpose of evaluating the differential operator $F(u)$ at any point along the boundary of the inner grid of the spatial domain. To calculate the values at these ghost points, we use the following five-point extrapolation formulae for three cases:

$$
\begin{aligned}
& x=L_{1}(\bullet): \quad u_{i, 0}=5 u_{i, 1}-10 u_{i, 2}+10 u_{i, 3}-5 u_{i, 4}+u_{i, 5}+\mathcal{O}\left(\Delta_{x}^{5}\right), \\
& y=L_{2}(\circ): \quad u_{0, j}=5 u_{1, j}-10 u_{2, j}+10 u_{3, j}-5 u_{4, j}+u_{5, j}+\mathcal{O}\left(\Delta_{y}^{5}\right) \text {, } \\
& x=L_{1}, y=L_{2}(\odot): \quad u_{0,0}=5 u_{1,1}-10 u_{2,2}+10 u_{3,3}-5 u_{4,4}+u_{5,5}+\mathcal{O}\left(\Delta_{x}^{5}\right) \\
& +\mathcal{O}\left(\Delta_{x}^{4} \Delta_{y}\right)+\mathcal{O}\left(\Delta_{x}^{3} \Delta_{y}^{2}\right)+\mathcal{O}\left(\Delta_{x}^{2} \Delta_{y}^{3}\right)+\mathcal{O}\left(\Delta_{x} \Delta_{y}^{4}\right)+\mathcal{O}\left(\Delta_{y}^{5}\right) .
\end{aligned}
$$

The extrapolation at the $x=K_{1}$ and $y=K_{2}$ boundaries and the remaining three corners is handled analogously. 


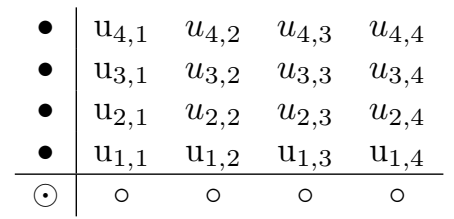

Figure 1: Example: evaluation of $F\left(u_{2,2}\right)$ using the $5 \times 5$-point computational stencil in the lower left corner of the computational domain; ghost points outside the computational domain at which values are extrapolated from the interior of the domain are marked by bullets $(\bullet, \circ, \odot)$, grid points on the boundary are set in Roman.

\section{Solving the high-order ADI scheme}

Starting from a given $U^{n-1}$, the ADI scheme (8) involves six approximation steps to obtain $U^{n}$, the solution at the next time level. The first approximation $Y_{0}$ can be solved for explicitly using the $5 \times 5$-point computational stencil derived in Section 5. The second approximation for our solution, denoted by $Y_{1}$, has to be solved for implicitly:

$$
Y_{1}=Y_{0}+\phi \Delta_{t}\left(F_{1}\left(Y_{1}\right)-F_{1}\left(U^{n-1}\right)\right) \quad \Longleftrightarrow \quad F_{1}\left(Y_{1}-U^{n-1}\right)=\frac{1}{\phi \Delta_{t}}\left(Y_{1}-Y_{0}\right) .
$$

We apply the fourth-order compact scheme established in Section 4 to solve (11). In matrix form we obtain

$$
A_{x}\left(Y_{1}-U^{n-1}\right)=B_{x}\left(\frac{1}{\phi \Delta_{t}}\left(Y_{1}-Y_{0}\right)\right)+d .
$$

Collecting unknown $Y_{1}$ terms on the left hand side and known terms $Y_{0}, U^{n-1}$ and $d$ on the right hand side we get

$$
\left(B_{x}-\phi \Delta_{t} A_{x}\right) Y_{1}=B_{x} Y_{0}-\phi \Delta_{t} A_{x} U^{n-1}-\phi \Delta_{t} d .
$$

To solve, we invert the tri-diagonal matrix $\left(B_{x}-\phi \Delta_{t} A_{x}\right)$. For the third step of the ADI scheme, we proceed analogously, and use the the high-order compact scheme presented in Section 4 to solve for $Y_{2}$ implicitly. The fourth, fifth and sixth step of the ADI scheme are performed analogously as the first, second and third steps, respectively.

Note that the matrix $\left(B_{x}-\phi \Delta_{t} A_{x}\right)$ appears twice in the scheme $(8)$, in the second and fifth step. Similarly, $\left(B_{y}-\phi \Delta_{t} A_{y}\right)$ appears in the third and the sixth step. Hence, using LU-factorisation, only two matrix inversions are necessary in each time step of scheme (8). Moreover, since the coefficients in the partial differential equation (5) do not depend on time, and the matrices are therefore constant, they can be LU-factorised before iterating in time to obtain a highly efficient algorithm.

The combination of the fourth-order spatial discretisation presented in Section 4 and 5 with the second-order time splitting (8) yields a high-order ADI scheme with order of consistency two in time and four in space. 


\section{Boundary conditions}

For the case of the Dirichlet conditions at $x=L_{1}$ and $x=K_{1}$ we impose

$$
\begin{aligned}
u\left(L_{1}, y_{j}, \tau_{k}\right) & =1-e^{r \tau+L_{1}}, & j & =1,2, \ldots, N, k=1,2, \ldots, \\
u\left(K_{1}, y_{j}, \tau_{k}\right) & =0, & j & =1,2, \ldots, N, k=1,2, \ldots
\end{aligned}
$$

Using the homogeneous Neumann conditions $(6 \mathrm{~d})$ and $6 \mathrm{e}$ which are correct in the limit $y \rightarrow \infty$ and $y \rightarrow 0$, respectively, at the (finite) boundaries $y=L_{2}>0$ and $y=K_{2}$ would result in a dominant error along these boundaries. Hence, we do not impose any boundary condition at these two boundaries but discretise the partial differential equation using the computational stencil from the interior. The values of the unknown on the boundaries are set by extrapolation from values in the interior. This introduces a numerical error, and it needs to be considered that the order of extrapolation should be high enough not to affect the overall order of accuracy. We refer to Gustafsson [20] to discuss the influence of the order of the approximation on the global convergence rate. We use the following extrapolation formulae:

$$
\begin{aligned}
& u_{i, 1}^{k}=5 u_{i, 2}^{k}-10 u_{i, 3}^{k}+10 u_{i, 4}^{k}-5 u_{i, 5}^{k}+u_{i, 6}^{k}+\mathcal{O}\left(\Delta_{y}^{6}\right), \\
& u_{i, N}^{k}=5 u_{i, N-1}^{k}-10 u_{i, N-2}^{k}+10 u_{i, N-3}^{k}-5 u_{i, N-4}^{k}+u_{i, N-5}^{k}+\mathcal{O}\left(\Delta_{y}^{6}\right) .
\end{aligned}
$$

\section{Sparse grid combination technique}

Due to the ADI splitting and the compactness of the finite difference discretisation in the implicit steps, the computational effort grows linearly with the number of unknowns, namely $\mathcal{O}(N \cdot M)$. In the following we use the so-called sparse grid combination technique to reduce the number of grid nodes and thus also the computational effort. Sparse grids go back to Smolyak [39], who used them for numerical integration. Zenger [42, Bungartz et al. 3] and Schiekofer [38] extended his idea and applied sparse grids to solve PDEs with finite element, finite volume and finite difference methods. These methods in general require hierarchical, tree-like data structures, which makes the data structure management more complicated than in the full grid case. With the help of the sparse grid combination technique [18 this problem can be overcome. Here, full tensor-based solutions are linearly combined to construct the sparse grid solution. This allows us to use standard full grid PDE solvers. Hence, this approach is very versatile and broadly applicable. Furthermore, each sub-solution can be computed independently, which makes it easily parallelisable.

The combination technique is based on the error splitting structure of the underlying numerical scheme. Let the numerical solution of the HO-ADI scheme be given by $u_{\boldsymbol{l}}$ with multi-index $\boldsymbol{l}=\left(l_{1}, l_{2}\right)$ and mesh widths $\Delta_{x}=2^{-l_{1}}\left(K_{1}-L_{1}\right)$, $\Delta_{y}=2^{-l_{2}}\left(K_{2}-L_{2}\right)$. We assume that our numerical scheme satisfies an error splitting structure of the form

$$
u-u_{l}=\Delta_{x}^{4} w_{1}\left(\Delta_{x}\right)+\Delta_{y}^{4} w_{2}\left(\Delta_{y}\right)+\Delta_{x}^{4} \Delta_{y}^{4} w_{1,2}\left(\Delta_{x}, \Delta_{y}\right),
$$

with functions $w_{1}, w_{2}, w_{1,2}$ bounded by some constant $C \in \mathbb{R}^{+}$. The mesh widths $\Delta_{x}$ and $\Delta_{y}$ are independent of one another. Since the error functions $w_{1}$ and $w_{2}$ only depend on either $\Delta_{x}$ or $\Delta_{y}$, we can subtract two solutions with the 
same mesh width in one coordinate direction, such that the error term cancels out. Exploiting this idea further leads to the combination technique

$$
u_{n}^{s}=\sum_{|l|_{1}=n+1} u_{l}-\sum_{|l|_{1}=n} u_{l} .
$$

Applying the error splitting from above, the lower order terms cancel out and we obtain

$$
\begin{gathered}
u_{n}^{s}=u+2^{-4(n+1)} R_{1} w_{1}\left(2^{-(n+1)} R_{1}\right)+2^{-2(n+1)\left(R_{2}\right)} w_{2}\left(2^{-(n+1)} R_{2}\right) \\
+2^{-4(n+1)} R_{1} R_{2} \sum_{i=0}^{n+1} w_{1,2}\left(2^{-i} R_{1}, 2^{-(n+1-i)} R_{2}\right) \\
\quad-2^{-4 n} R_{1} R_{2} \sum_{i=0}^{n} w_{1,2}\left(2^{-i} R_{1}, 2^{-(n-i)} R_{2}\right),
\end{gathered}
$$

where $R_{1}=K_{1}-L_{1}$ and $R_{2}=K_{2}-L_{2}$. As $w_{1}, w_{2}$ and $w_{1,2}$ are bounded by $C$ the pointwise error is given by

$$
\left|u_{n}^{s}-u\right|=\mathcal{O}\left(n 2^{-4 n}\right),
$$

which is equivalent to

$$
\left|u_{n}^{s}-u\right|=\mathcal{O}\left(\Delta^{4} \log _{2}\left(\Delta^{-1}\right)\right)
$$

for $\Delta=2^{-n}$. We observe that the error of the sparse grid combination technique is deteriorated by a factor of $\log _{2}\left(\Delta^{-1}\right)$ compared to the fourth-order full grid solution.

Figure 2 shows the two-dimensional grid hierarchy at levels $n=0, \ldots, 4$. The sparse grid in two dimensions at level $n$ consists of sub-grids, whose sum of refinement levels fulfils $|\boldsymbol{l}|_{1}=n$. Hence the number of grid points on each sub-grid grows with $\mathcal{O}\left(2^{n}\right)$. As the number of grids increases with $\mathcal{O}(n)$, this leads to $\mathcal{O}\left(n 2^{n}\right)$ nodes in the sparse grid. Let $\Delta=2^{-n}$, then this results in $\mathcal{O}\left(\Delta^{-1} \log _{2}\left(\Delta^{-1}\right)\right)$ grid points compared to $\mathcal{O}\left(\Delta^{-2}\right)$ nodes in the full grid. Thus we are able to reduce the number of grid nodes significantly while maintaining a high accuracy.

It should be noted that for larger $n$ the combination technique as introduced above involves solutions on grids which violate the assumption of moderate cell aspect ratios which may lead to reduced accuracy and potential instability of the scheme due to the extreme distortion of the grid. This aspect of the combination technique is of general nature and not specific to our scheme. A usual remedy would be to exclude solutions on extremely distorted grids in 12 . For further details we refer to the pertinent literature on sparse grids.

\section{Numerical experiments}

In this section we test the proposed sparse grid high-order ADI scheme. Beside the accuracy of the full grid solution we are also interested in the efficiency of the combined sparse grid solution.

It is well known that due to the non-smooth nature of the payoff function in option pricing problems one cannot expect to see higher-order in practice 


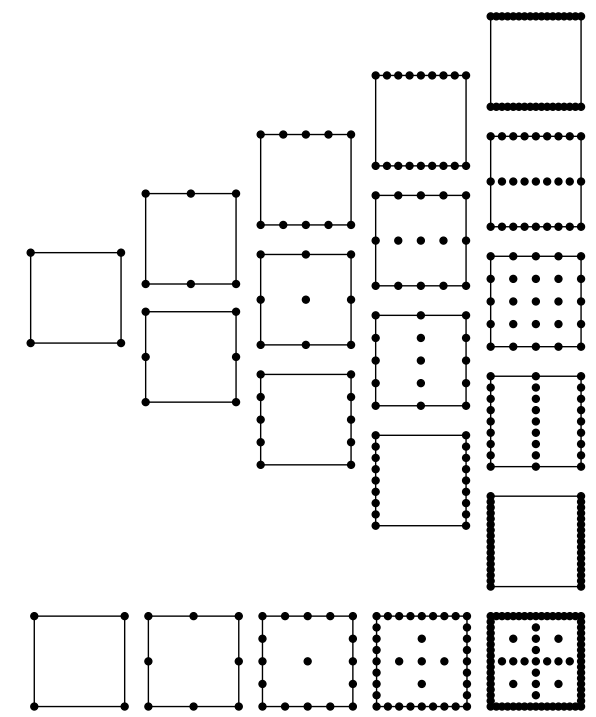

Figure 2: Sub-grids and sparse grid for $n=0, \ldots, 4$.

35. Some form of smoothing has to be applied to the initial condition. In 29] suitable smoothing operators are identified in Fourier space. Since the order of convergence of our high-order compact scheme is four, we could use the smoothing operator $\Phi_{4}$ as in [14, given by its Fourier transformation

$$
\hat{\Phi}_{4}(\omega)=\left(\frac{\sin \left(\frac{\omega}{2}\right)}{\frac{\omega}{2}}\right)^{4}\left[1+\frac{2}{3} \sin ^{2}\left(\frac{\omega}{2}\right)\right] .
$$

This leads to the smooth initial condition determined by

$$
\tilde{u}_{0}(x, y)=\int_{-3 h}^{3 h} \int_{-3 h}^{3 h} \Phi_{4}\left(\frac{\tilde{x}}{h}\right) \Phi_{4}\left(\frac{\tilde{y}}{h}\right) u_{0}(x-\tilde{x}, y-\tilde{y}) d \tilde{x} d \tilde{y}
$$

for any stepsize $h>0$, where $u_{0}$ is the original initial condition and $\Phi_{4}(x)$ denotes the Fourier inverse of $\hat{\Phi}_{4}(\omega)$, see [29]. As $h \rightarrow 0$, the smooth initial condition $\tilde{u}_{0}$ tends towards the original initial condition $u_{0}$ and the approximation of the smoothed problem tends towards the true solution. For our numerical experiments we use this smoothing operator which has already been applied successfully to option pricing problems in [14].

A numerical solution computed on a grid with $\Delta_{x}=\Delta \cdot\left(K_{1}-L_{1}\right), \Delta_{y}=$ $\Delta \cdot\left(K_{2}-L_{2}\right)$ and time step $\Delta_{t}=5 \cdot \Delta^{2}$ serves as a reference solution, where $\Delta=2^{-8}$. Since the accuracy of option prices close to the strike price is of highest interest from a practitioner's point of view, we compute the maximum absolute error in the region $[0.5 E, 2 E] \times[0.05,1]$. The grid parameters of the computational domain are chosen to be $L_{1}=-5, K_{1}=1.5, L_{2}=0.05$ and $K_{2}=2.5$. The parameters of the ADI method are $\psi=1 / 2$ and $\phi=1 / 2$, cf. Section 3 . The full grid solution is computed with step sizes $\Delta_{x}=\Delta \cdot\left(K_{1}-L_{1}\right)$, $\Delta_{y}=\Delta \cdot\left(K_{2}-L_{2}\right)$ and $\Delta_{t}=5 \cdot \Delta^{2}$ with $\Delta=2^{-n}$, while the sparse grid solution $u_{n}^{s}$ is constructed according to definition $(12)$. In order to avoid instabilities due 


\begin{tabular}{ll}
\hline Parameter & Value \\
\hline Strike price & $E=100$ \\
Time to maturity & $T=1$ \\
Interest rate & $r=0.05$ \\
Volatility of volatility & $v=0.1$ \\
Mean reversion speed & $\kappa=2$ \\
Long run mean of volatility & $\theta=0.1$ \\
Correlation & $\rho=-0.5$ \\
Stochastic volatility drift parameter & $\alpha=0.5$ \\
Stochastic volatility diffusion parameter & $\beta=0.5$ \\
\hline
\end{tabular}

Figure 3: Parameters used in the numerical experiments.

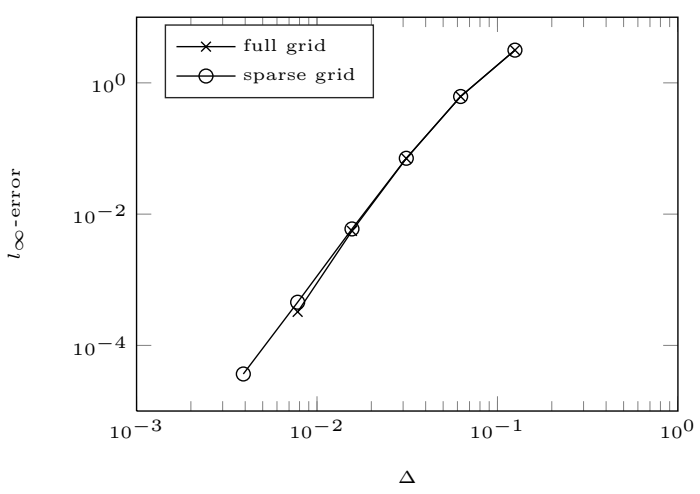

Figure 4: Error decay of the full grid for $n=3,4, \ldots, 7$ and sparse grid combination technique for $n=6,7, \ldots, 11$.

to the extreme distortion of the grid we neglect grids within the combination technique, where $l_{i} \leq 2$ for $i=1,2$. Thus, the finest resolution in one of the sub-grids along one coordinate direction is given by $\Delta=2^{-(n-3)}$.

We compare the performance of the high-order ADI scheme in the full and sparse grid case for a European put option with the parameters given in Table 3 . Figure 4 shows the maximum error plotted versus the grid resolution $\Delta$ for both cases. The fourth-order compact finite difference scheme achieves an estimated numerical convergence order of 3.33, the error of the sparse grid solution decays slightly slower due to the logarithmic factor in (13).

To illustrate the computational efficiency we compare the run-time to the accuracy in Figure 5 for both approaches. We confirm that, as the mesh width decreases, the lower number of employed grid nodes in the sparse grid method outweighs its slightly lower convergence rate. The serial implementation of the combination technique outperforms the full grid solver in the high accuracy region, reducing the computational time by about an order of magnitude, while achieving a similar accuracy. 


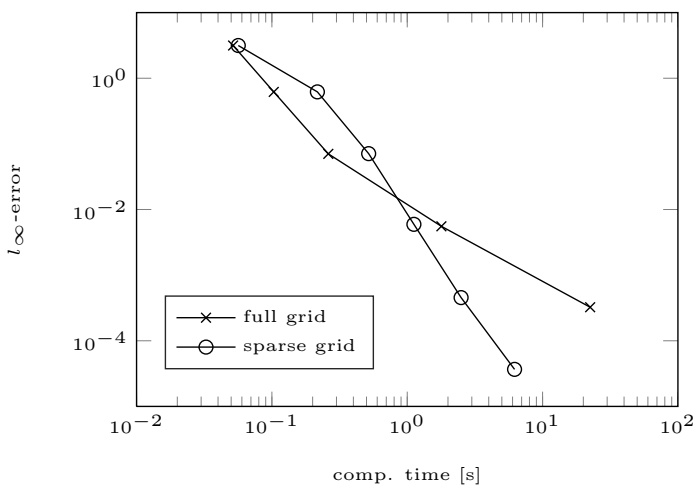

Figure 5: Error versus computational time for the full grid for $n=3,4, \ldots, 7$ and sparse grid combination technique for $n=6,7, \ldots, 11$.

\section{Acknowledgement}

BD acknowledges support by the Leverhulme Trust research project grant 'Novel discretisations for higher-order nonlinear PDE' (RPG-2015-69). CH is supported by the European Union in the FP7-PEOPLE-2012-ITN Program under Grant Agreement Number 304617 (FP7 Marie Curie Action, Project Multi-ITN STRIKE - Novel Methods in Computational Finance). Further CH acknowledges partial support from the bilateral German-Spanish Project HiPeCa - High Performance Calibration and Computation in Finance, Programme Acciones Conjuntas Hispano-Alemanas financed by DAAD. JM has been supported in part by a studentship under the EPSRC Doctoral Training Partnership (DTP) scheme (grant number EP/L505109/1).

\section{References}

[1] R.M. Beam and R.F. Warming, Alternating direction implicit methods for parabolic equations with a mixed derivative, SIAM J. Sci. Stat. Comput. $\mathbf{1}(1), 1980$.

[2] E. Benhamou, E. Gobet and M. Miri. Time dependent Heston model, SIAM J. Finan. Math. 1, 289-325, 2010.

[3] H. Bungartz and M. Griebel. Sparse grids, Acta Numer. 13, 1-123, 2004.

[4] P. Christoffersen, K. Jacobs and K. Mimouni. Volatility dynamics for the S\&P500: evidence from realized volatility, daily returns, and option prices. Review of Financial Studies 23, 3141-3189, 2010.

[5] N. Clarke and K. Parrott. Multigrid for American option pricing with stochastic volatility. Appl. Math. Finance 6(3), 177-195, 1999.

[6] J. Douglas, Alternating direction methods for three space variables, Numer. Math., 4, 41-63, 1962. 
[7] J. Douglas and J.E. Gunn, A general formulation of alternating direction methods. I. Parabolic and hyperbolic problems, Numer. Math. 6, 428-453, 1964.

[8] J. Duan. The GARCH option pricing model. Math. Finance 5(1), 13-32, 1995.

[9] B. Düring and M. Fournié. High-order compact finite difference scheme for option pricing in stochastic volatility models. J. Comput. Appl. Math. 236(17), 4462-4473, 2012.

[10] B. Düring, M. Fournié and C. Heuer. High-order compact finite difference schemes for option pricing in stochastic volatility models on non-uniform grids. J. Comput. Appl. Math. 271(18), 247-266, 2014.

[11] B. Düring, M. Fournié and A. Jüngel. Convergence of a high-order compact finite difference scheme for a nonlinear Black-Scholes equation. Math. Mod. Num. Anal. 38(2), 359-369, 2004.

[12] B. Düring, M. Fournié and A. Jüngel. High-order compact finite difference schemes for a nonlinear Black-Scholes equation. Intern. J. Theor. Appl. Finance 6(7), 767-789, 2003.

[13] B. Düring, M. Fournié and A. Rigal. High-order ADI schemes for convection-diffusion equations with mixed derivative terms. In: Spectral and High Order Methods for Partial Differential Equations - ICOSAHOM'12, M. Azaez et al. (eds.), pp. 217-226, Lecture Notes in Computational Science and Engineering 95, Springer, Berlin, Heidelberg, 2013.

[14] B. Düring and C. Heuer. High-order compact schemes for parabolic problems with mixed derivatives in multiple space dimensions. SIAM J. Numer. Anal. 53(5), 2113-2134, 2015.

[15] B. Düring. Asset pricing under information with stochastic volatility. Rev. Deriv. Res. 12(2), 141-167, 2009.

[16] B. Düring and J. Miles. High-order ADI scheme for option pricing in stochastic volatility models. J. Comput. Appl. Math., 2016, http://dx. doi.org/10.1016/j.cam.2016.09.040.

[17] M. Fournié and A. Rigal. High order compact schemes in projection methods for incompressible viscous flows, Commun. Comput. Phys. 9(4), 9941019, 2011.

[18] M. Griebel, M. Schneider and C. Zenger. A Combination Technique for the Solution of Sparse Grid Problems. IMACS Elsevier, Iterative Methods in Linear Algebra, 16:263-281, 1992.

[19] M.M. Gupta, R.P. Manohar and J.W. Stephenson, A single cell high-order scheme for the convection-diffusion equation with variable coefficients, Int. J. Numer. Methods Fluids, 4, 641-651, 1984.

[20] B. Gustafsson. The convergence rate for difference approximation to general mixed initial-boundary value problems. SIAM J. Numer. Anal. 18(2), 179190, 1981. 
[21] C. Hendricks, M. Ehrhardt and M. Günther, High-order ADI schemes for diffusion equations with mixed derivatives in the combination technique, Appl. Numer. Math. 101, 36-52, 2016.

[22] S.L. Heston. A closed-form solution for options with stochastic volatility with applications to bond and currency options. Review of Financial Studies 6(2), 327-343, 1993.

[23] K.J. in't Hout and S. Foulon. ADI finite difference schemes for option pricing in the Heston model with correlation. Int. J. Numer. Anal. Mod. 7, 303-320, 2010.

[24] K.J. int Hout and B.D. Welfert. Stability of ADI schemes applied to convection-diffusion equations with mixed derivative terms. Appl. Num. Math. 57, 19-35, 2007.

[25] W. Hundsdorfer and J.G. Verwer, Numerical solution of time-dependent advection-diffusion-reaction equations, Springer Series in Computational Mathematics, 33, Springer-Verlag, Berlin, 2003.

[26] W. Hundsdorfer, Accuracy and stability of splitting with stabilizing corrections, Appl. Num. Math. 42, 213-233, 2002.

[27] N. Hilber, A. Matache and C. Schwab. Sparse wavelet methods for option pricing under stochastic volatility. J. Comput. Financ. 8(4), 1-42, 2005.

[28] S. Ikonen and J. Toivanen. Efficient numerical methods for pricing American options under stochastic volatility. Numer. Methods Partial Differential Equations 24(1), 104-126, 2008.

[29] H.O. Kreiss, V. Thomée and O. Widlund. Smoothing of initial data and rates of convergence for parabolic difference equations. Comm. Pure Appl. Math. 23, 241-259, 1970.

[30] W. Liao and A.Q.M. Khaliq. High-order compact scheme for solving nonlinear Black-Scholes equation with transaction cost. Int. J. Comput. Math. 86(6), 1009-1023, 2009.

[31] D. Lanser, J, Blom and J. Verwer. Time integration of the shallow water equations in spherical geometry. J. Comp. Phys. 171, 373-393, 2001.

[32] A.L. Lewis. Option valuation under stochastic volatility. Finance Press, Newport Beach, CA, 2000.

[33] A.R. Mitchell and G. Fairweather, Improved forms of the alternating direction methods of Douglas, Peaceman, and Rachford for solving parabolic and elliptic equations, Numer. Math. 6, 285-292, 1964.

[34] D.W. Peaceman and H.H. Rachford Jr., The numerical solution of parabolic and elliptic differential equations, J. Soc. Ind. Appl. Math. 3, 28-41, 1959.

[35] D. M. Pooley, K. R. Vetzal and P. A. Forsyth. Convergence remedies for non-smooth payoffs in option pricing. J. Comput. Financ. 6(4), 25-40, 2003. 
[36] M.J. Ruijter and C.W. Oosterlee. Two-dimensional Fourier cosine series expansion method for pricing financial options, SIAM J. Sci. Comp. 34(5), 642-671, 2012.

[37] A. Rigal, Schémas compacts d'ordre élevé: application aux problèmes bidimensionnels de diffusion-convection instationnaire I, C.R. Acad. Sci. Paris. Sr. I Math. 328, 535-538, 1999.

[38] T. Schiekofer, Die Methode der Finiten Differenzen auf dünnen Gittern zur Lösung elliptischer und parabolischer partieller Differentialgleichungen, PhD thesis, Universität Bonn, 1999.

[39] S. Smolyak, Quadrature and interpolation formulas for tensor products of certain classes of functions, Dokl. Akad. Nauk SSSR 148, 1042-1045, 1963.

[40] W.F. Spotz and C.F. Carey. Extension of high-order compact schemes to time-dependent problems. Numer. Methods Partial Differential Equations 17(6), 657-672, 2001.

[41] D. Tavella and C. Randall. Pricing financial instruments: the finite difference method. John Wiley \& Sons, 2000.

[42] C. Zenger. Sparse grids. Technical report, Institut für Informatik, Technische Universität München, Oct. 1990.

[43] W. Zhu and D.A. Kopriva. A spectral element approximation to price European options with one asset and stochastic volatility. J. Sci. Comput. 42(3), 426-446, 2010.

[44] R. Zvan, P.A. Forsyth and K.R. Vetzal. Penalty methods for American options with stochastic volatility. J. Comp. Appl. Math. 91(2), 199-218, 1998. 